\title{
A composite outcome for neonatal cardiac surgery research
}

\author{
Ryan J. Butts, MD, ${ }^{\text {a }}$ Mark A. Scheurer, MD, ${ }^{\mathrm{a}}$ Sinai C. Zyblewski, MD, ${ }^{\mathrm{a}}$ Amy E. Wahlquist, MS, \\ Paul J. Nietert, PhD, ${ }^{b}$ Scott M. Bradley, MD, ${ }^{c}$ Andrew M. Atz, MD, and Eric M. Graham, MD ${ }^{a}$
}

Objective: The objective of this study was to determine whether a composite outcome, derived of objective signs of inadequate cardiac output, would be associated with other important measures of outcomes and therefore be an appropriate end point for clinical trials in neonatal cardiac surgery.

\begin{abstract}
Methods: Neonates $(\mathrm{n}=76)$ undergoing cardiac operations requiring cardiopulmonary bypass were prospectively enrolled. Patients were defined to have met the composite outcome if they had any of the following events before hospital discharge: death, the use of mechanical circulatory support, cardiac arrest requiring chest compressions, hepatic injury ( 2 times the upper limit of normal for aspartate aminotransferase or alanine aminotransferase), renal injury (creatinine $>1.5 \mathrm{mg} / \mathrm{dL}$ ), or lactic acidosis (an increasing lactate $>5 \mathrm{mmol} / \mathrm{L}$ in the postoperative period). Associations between the composite outcome and the duration of mechanical ventilation, intensive care unit stay, hospital stay, and total hospital charges were determined.
\end{abstract}

Results: The median age at the time of surgery was 7 days, and the median weight was $3.2 \mathrm{~kg}$. The composite outcome was met in $39 \%$ of patients (30/76). Patients who met the composite outcome compared with those who did not had a longer duration of mechanical ventilation ( 4.9 vs 2.9 days, $P<.01$ ), intensive care unit stay ( 8.8 vs 5.7 days, $P<.01$ ), hospital stay ( 23 vs 12 days, $P<.01$ ), and increased hospital charges ( $\$ 258,000$ vs $\$ 170,000$, $P<.01)$. In linear regression analysis, controlling for surgical complexity, these differences remained significant $\left(R^{2}=0.29-0.42, P<.01\right)$.

Conclusions: The composite outcome is highly associated with important early operative outcomes and may serve as a useful end point for future clinical research in neonates undergoing cardiac operations. (J Thorac Cardiovasc Surg 2014;147:428-33)

Advancements in surgical techniques and refinements in perioperative management have led to significant improvements in survival after neonatal cardiac surgery, yet significant morbidity persists. ${ }^{1-3}$ Accordingly, research has shifted from not only improving survival but also reducing morbidity. ${ }^{4}$ However, research in pediatric heart disease is confronted by barriers, including small numbers of subjects with a particular congenital heart defect at any one center, differences in treatment approaches resulting in a lack of therapeutic equipoise, and lack of validated outcome measures. Multi-institutional collaboration, such as

From the Division of Cardiology, ${ }^{\mathrm{a}}$ Department of Pediatrics, Division of Biostatistics and Epidemiology, ${ }^{\mathrm{b}}$ Department of Medicine, and Division of Cardiothoracic Surgery, ${ }^{\mathrm{c}}$ Department of Surgery, Medical University of South Carolina, Charleston, SC

This study was supported in part by a Career Development Award from the American College of Cardiology Foundation/Pfizer Scholarship (to Dr Graham) and by Award Number UL1RR029882 from the National Center for Research Resources. The content is solely the responsibility of the authors and does not necessarily represent the official views of the National Center for Research Resources or the National Institutes of Health.

Disclosures: Authors have nothing to disclose with regard to commercial support.

Received for publication Oct 15, 2012; revisions received Jan 17, 2013; accepted for publication March 8, 2013; available ahead of print April 15, 2013.

Address for reprints: Ryan J. Butts, MD, Medical University of South Carolina, 165

Ashley Ave, MSC 915, Charleston, SC 29425 (E-mail: butts@musc.edu). 0022-5223/\$0.00

Published by Elsevier Inc. on behalf of The American Association for Thoracic Surgery

http://dx.doi.org/10.1016/j.jtcvs.2013.03.013 the National Heart, Lung, and Blood Institute-funded Pediatric Heart Network, and registries have been developed to improve sample size and facilitate scientific investigation. ${ }^{5}$ However, validation of outcomes remains relatively unexplored. In congenital cardiac surgery, commonly used outcomes include mortality, duration of mechanical ventilation, intensive care unit (ICU) stay, and hospital stay. ${ }^{6,7}$ In the neonatal population, these outcomes often can be problematic because mortality is infrequent, and the other outcomes often have a wide range or large standard deviation resulting in inadequately powered studies or the need for a large sample size. Many investigators have used surrogate outcome measures, such as low cardiac output syndrome, inotropic score, and vasoactive inotropic score. ${ }^{6-8}$ Despite the intuitive nature of these outcomes, a recent report demonstrated that low cardiac output syndrome had a poor association and vasoactive inotropic score had weak correlations with other clinically important outcomes in neonates undergoing cardiac operations. ${ }^{9}$ This highlights the need for further investigation into the validation of superior surrogate outcome measures in neonatal cardiac surgery.

An ideal early outcome measure would be easily measured, reproducible, and, most important, independently correlated with other measures of short- and long-term outcomes. Composite outcomes, which combine several components into a single measure, have gained popularity in the 


$$
\begin{aligned}
& \text { Abbreviations and Acronyms } \\
& \begin{aligned}
\text { ALT } & =\text { alanine aminotransferase } \\
\text { AST } & =\text { aspartate aminotransferase } \\
\text { CI } & =\text { confidence interval } \\
\text { CPB } & =\text { cardiopulmonary bypass } \\
\text { CPR } & =\text { cardiopulmonary resuscitation } \\
\text { ECMO } & =\text { extracorporeal membrane oxygenation } \\
\text { ICU } & =\text { intensive care unit } \\
\text { IQR } & =\text { interquartile range } \\
\text { RACHS- } & \text { Risk Adjustment for Congenital Heart } \\
1 & \text { Surgery } 1
\end{aligned}
\end{aligned}
$$

scientific community. ${ }^{10}$ The advantages supporting the use of a composite outcome are that it increases statistical efficiency because of higher event rates, which reduces sample size requirement, cost, and time, and that it allows investigators to avoid an arbitrary choice between several important outcomes. ${ }^{11}$ These advantages have resulted in the widespread use of composite outcomes in clinical trials, including pediatric heart disease. ${ }^{12-14}$ The objective of this study was to use a standardized composite outcome for neonatal cardiac surgery in a well-characterized cohort and correlate this composite outcome with other important early postoperative outcomes.

\section{MATERIALS AND METHODS}

The study is a secondary analysis of a prospective randomized controlled trial comparing preoperative glucocorticoid therapy in 76 neonates (ClinicalTrials.gov Identifier NCT00934843). ${ }^{15}$ The study was approved by the Medical University of South Carolina's Institutional Review Board, and informed written consent was obtained from the parent or legal guardian of all participants.

\section{Composite Outcome}

The individual components of the composite outcome were composed of clinical and laboratory signs of inadequate tissue oxygen delivery or circulatory collapse. Six components were chosen because each represents important early clinical outcomes, often resulting from low cardiac output, and have been associated with poor outcomes in other studies. ${ }^{16-21}$ The components include death, circulatory collapse requiring chest compressions (cardiopulmonary resuscitation [CPR]), mechanical circulatory support (extracorporeal membrane oxygenation [ECMO]), hepatic insufficiency, renal insufficiency, and lactic acidosis. Hepatic injury was defined as aspartate aminotransferase (AST) or alanine aminotransferase (ALT) greater than 2 times the upper limit of normal (normal range, AST 8-40 IU/L; hepatic injury, AST >80 IU/L; normal range, ALT 7-40 IU/L; hepatic injury, ALT $>80 \mathrm{IU} / \mathrm{L}$ ). Elevated AST within the first 24 postoperative hours was not considered hepatic insufficiency because AST elevation was likely a result of hemolysis related to cardiopulmonary bypass (CPB). ${ }^{22}$ Renal injury was defined by an increase in creatinine during admission greater than 2 times the upper limit of normal (normal range, 0.3-0.7; renal injury, $>1.5 \mathrm{mg} / \mathrm{dL}$ ). Lactic acidosis was defined as an increasing arterial lactate concentration that reached more than $5 \mathrm{mmol} / \mathrm{dL}$ postoperatively. ${ }^{16,23}$ Patients who experienced any of the 6 components after cardiac surgery and before hospital discharge were considered to have met the composite outcome.
Liver function panels were ordered as part of the study protocol on arrival to the ICU and 36 hours after arrival. Additional liver function tests were obtained as clinically indicated and analyzed for the composite outcome. Serum creatinine was measured on arrival to the ICU from the operating room and then daily for a minimum of 2 days. It was common practice during the study period to have creatinine levels checked daily while in the ICU if arterial or central venous access was present; however, this practice was not required by the study protocol. Lactate levels were obtained via arterial catheters and obtained on arrival to the ICU and at 1 hour, 2 hours, 4 hours, 8 hours, 12 hours, and 24 hours postoperatively at a minimum and more often as clinically indicated.

\section{Study Population}

All inpatient neonates (aged $\leq 30$ days) scheduled to undergo cardiac surgery involving CPB from July 2007 to July 2009 were eligible for this study. Exclusion criteria included prematurity (defined as $\leq 36$ weeks gestational age at the time of surgery), previous treatment with or contraindication to steroid therapy, or preoperative use of mechanical circulatory support or active resuscitation at the time of proposed randomization. $\mathrm{Pa}$ tients were randomly assigned to preoperative placebo and intraoperative methylprednisolone (1 dose) or preoperative and intraoperative methylprednisolone ( 2 doses). Details on the study population have been reported. $^{15}$

\section{Data Analysis}

The characteristics of the patients who met the composite outcome were compared with those who did not with respect to surgical complexity by the Risk Adjustment for Congenital Heart Surgery 1 (RACHS-1) classification, ${ }^{24}$ age at surgery, weight at surgery, length of CPB, use of aprotinin, or delayed sternal closure. Continuous data were compared using the Wilcoxon rank-sum test; categoric data were analyzed with the chi-square test. Patients who met the composite outcome were compared with those who did not with respect to duration of postoperative mechanical ventilation, ICU stay, hospital stay, and hospital charges using the Wilcoxon rank-sum test.

To explore the associations between meeting more than 1 criteria or meeting the more clinically significant criteria and outcomes, patients were further separated into 3 groups based on the number of composite outcome criteria met: none, only 1 , and more than 1 . Patients were further classified as meeting 1 of the clinical criteria (CPR, ECMO, death), laboratory criteria only (hepatic or renal injury, lactic acidosis), or no criteria. Comparisons were made between groups using the Kruskal-Wallis test for nonparametric data. Because patients who die in the hospital often have more extensive resource use, sensitivity analyses were performed to determine whether findings changed significantly when those patients $(n=2)$ were excluded.

To perform linear regression analysis, postoperative outcomes were logtransformed to create parametric distribution. Surgical complexity was controlled in the regressions analysis by dichotomizing patients with a RACHS- 1 class of 5 or 6 and patients with a RACHS- 1 class of 2 to 4 . An alpha level less than 0.05 was considered statistically significant.

\section{RESULTS}

All 76 patients had complete records for review and were included in the analysis. Patient demographics, cardiac diagnoses, surgical procedures performed, and intraoperative parameters have been described. ${ }^{15}$ Median gestational age was 39 weeks (interquartile range [IQR], 38-39.3). Median weight at surgery was $3.2 \mathrm{~kg}$ (IQR, 2.9-3.5). Diagnostic classification based on RACHS-1 class is shown in Table 1.

Of the 76 patients, 30 (39\%) met the composite outcome. There were 2 deaths before hospital discharge, resulting in 
TABLE 1. Diagnostic classification

\begin{tabular}{lc}
\hline RACHS-1 category & $\mathbf{N = 7 6}$ \\
\hline 2 & $6(8 \%)$ \\
3 & $25(33 \%)$ \\
4 & $25(33 \%)$ \\
5 & $1(1 \%)$ \\
6 & $19(25 \%)$ \\
Corrective surgery & $43(57 \%)$ \\
\hline
\end{tabular}

RACHS-1, Risk-Adjusted Classification for Congenital Heart Surgery.

a $97 \%$ hospital survival. ECMO was used postoperatively in 4 patients (for an inability to wean from CPB in 2 and for low cardiac output in 2); all survived. Cardiac arrest requiring chest compressions occurred in 3 patients, and all were successfully resuscitated without the use of mechanical support. A serum lactate that increased in the postoperative period and that was greater than $5 \mathrm{mmol} / \mathrm{L}$ was present in 14 patients. Hepatic injury occurred in 17 patients, and 4 patients had renal injury. More than 1 criterion for the composite outcome was present in 10 patients.

Preoperative and intraoperative characteristics of those who met the composite outcome compared with those who did not are shown in Table 2. The group that met the composite outcome had a higher RACHS-1 categorization than the group that did not meet the composite outcome. The groups were otherwise similar with respect to age and weight at time of surgery, CPB time, use of aprotinin, corrective versus palliative surgery, and use of delayed sternal closure. Between steroid treatment groups, there were no differences in meeting the composite outcome $(49 \% 1$ dose vs $51 \% 2$ doses, $P=.8$ ).

Patients who met the composite outcome had longer median hospital stay by 11 days, ICU stay by 3.1 days, mechanical ventilation by 2 days, and higher median hospital charges by $\$ 88,000$ compared with those who did not meet the composite outcome (Table 3). Linear regression analysis showed that after controlling for RACHS-1 classification, patients who met the composite outcome had an $83 \%$ longer hospital stay $(95 \%$ confidence interval $[\mathrm{CI}]$, $\left.42-137 ; R^{2}=0.42 ; P<.01\right), 88 \%$ longer ICU stay $(95 \%$

TABLE 2. Patient characteristics and composite outcome

\begin{tabular}{lccc}
\hline & \multicolumn{2}{c}{ Composite outcome } & \\
\cline { 2 - 3 } & \multicolumn{1}{c}{ Yes $(\mathbf{n}=\mathbf{3 0})$} & No $(\mathbf{n}=\mathbf{4 6})$ & P value \\
\hline RACHS-1 class & $4(4-6)$ & $3(3-4)$ & .01 \\
Age (d) & $7(5-12)$ & $7(6-8)$ & .95 \\
Weight at surgery (kg) & $3.3(2.8-3.6)$ & $3.2(2.9-3.5)$ & .89 \\
Delayed sternal closure, n (\%) & $19(63 \%)$ & $19(41 \%)$ & .06 \\
CPB (min) & $162(123-215)$ & $155(128-171)$ & .28 \\
Aprotinin use, n (\%) & $17(57 \%)$ & $17(37 \%)$ & .15 \\
Corrective surgery, n (\%) & $16(53 \%)$ & $27(59 \%)$ & .82 \\
\hline
\end{tabular}

RACHS-1, Risk-Adjusted Classification for Congenital Heart Surgery; $C P B$, cardiopulmonary bypass. *Continuous data expressed in median (IQR); Wilcoxon rank-sum test performed.
TABLE 3. Composite outcome and postoperative outcomes

\begin{tabular}{llcl} 
& \multicolumn{2}{c}{ Composite outcome } & \\
\cline { 2 - 3 } & Yes $(\mathbf{n}=\mathbf{3 0})$ & No $(\mathbf{n}=\mathbf{4 6})$ & P value \\
\hline Hospital stay (d) & $23(16-39)$ & $12(9-22)$ & $<.01$ \\
ICU stay (d) & $8.8(6.3-19)$ & $5.7(4.1-7)$ & $<.01$ \\
Mechanical ventilation (d) & $4.9(3.8-12.8)$ & $2.9(1.9-3.9)$ & $<.01$ \\
Hospital charges (\$1000) & $258(188-403)$ & $170(137-211)$ & $<.01$ \\
\hline Values displayed are medians (IQR). ICU, Intensive care unit.
\end{tabular}

CI, 42-150; $\left.R^{2}=0.34 ; P<.01\right), 105 \%$ longer time on mechanical ventilation $\left(95 \% \mathrm{CI}, 42-197 ; R^{2}=0.29 ; P<.01\right)$, and $52 \%$ higher total hospital charges $(95 \%$ CI, 25-84; $\left.R^{2}=0.33 ; P<.01\right)$.

Patients who met more than 1 criteria for the composite outcome had longer median hospital stay by 20.5 days, ICU stay by 11.6 days, mechanical ventilation by 7.8 days, and higher median hospital charges by $\$ 193,000$, compared with those who met 1 criteria (Table 4). Patients who met 1 criteria had longer hospital stay, ICU stay, and mechanical ventilation, and higher hospital charges compared with those who met no criteria (Table 4 and Figure 1).

Patients who met 1 of the clinical criteria of death, ECMO, or CPR had longer median hospital stay, ICU stay, and mechanical ventilation, as well as higher medial hospital charges, compared with those who met only laboratory criteria (Table 5 and Figure 2). These differences persisted when comparing patients who met the laboratory criteria only with those who met no criteria (Table 5 and Figure 2). After excluding the 2 patients who died, all associations shown in Tables 3,4 , and 5 remained relatively unchanged and statistically significant $(P<.01)$.

\section{DISCUSSION}

The results of this study demonstrate that the composite outcome is both reasonably prevalent and highly associated with important early operative outcomes and thus may serve as a useful end point for future clinical research in neonates undergoing cardiac operations with CPB. The components of the composite outcome were selected on the basis of

TABLE 4. Number of composite outcome criteria met and postoperative outcomes

\begin{tabular}{llllll}
\hline & \multicolumn{3}{c}{ Composite outcome } & \\
\cline { 2 - 3 } & \multicolumn{2}{c}{ Yes } & No & $\boldsymbol{P}$ \\
\cline { 2 - 3 } & \multicolumn{1}{c}{$\mathbf{1}(\mathbf{n}=\mathbf{1 0})$} & $\mathbf{1}(\mathbf{n}=\mathbf{2 0})$ & $\mathbf{( n = 4 6 )}$ & value \\
\hline Hospital stay (d) & $41.5(24.0-60.0)$ & $21.0(16.5-36.0)$ & $12.0(9.0-22.0)$ & $<.01$ \\
ICU stay (d) & $19.4(10.8-36.0)$ & $7.8(6.1-10.3)$ & $5.7(4.1-7.0)$ & $<.01$ \\
Mechanical & $12.4(7.0-28.0)$ & $4.6(3.4-5.4)$ & $2.9(1.9-3.9)$ & $<.01$ \\
$\quad \begin{array}{lllll}\text { ventilation (d) } \\
\text { Hospital charges }\end{array}$ & $418(267-506)$ & $225(184-262)$ & $170(137-211)$ & $<.01$ \\
$\quad(\$ 1000)$ & & & & \\
\hline
\end{tabular}

Values displayed are medians (IQR), $>1=$ patients who met $\geq 2$ composite outcome criteria, 1 = patients who met 1 composite outcome criteria. $I C U$, Intensive care unit. 

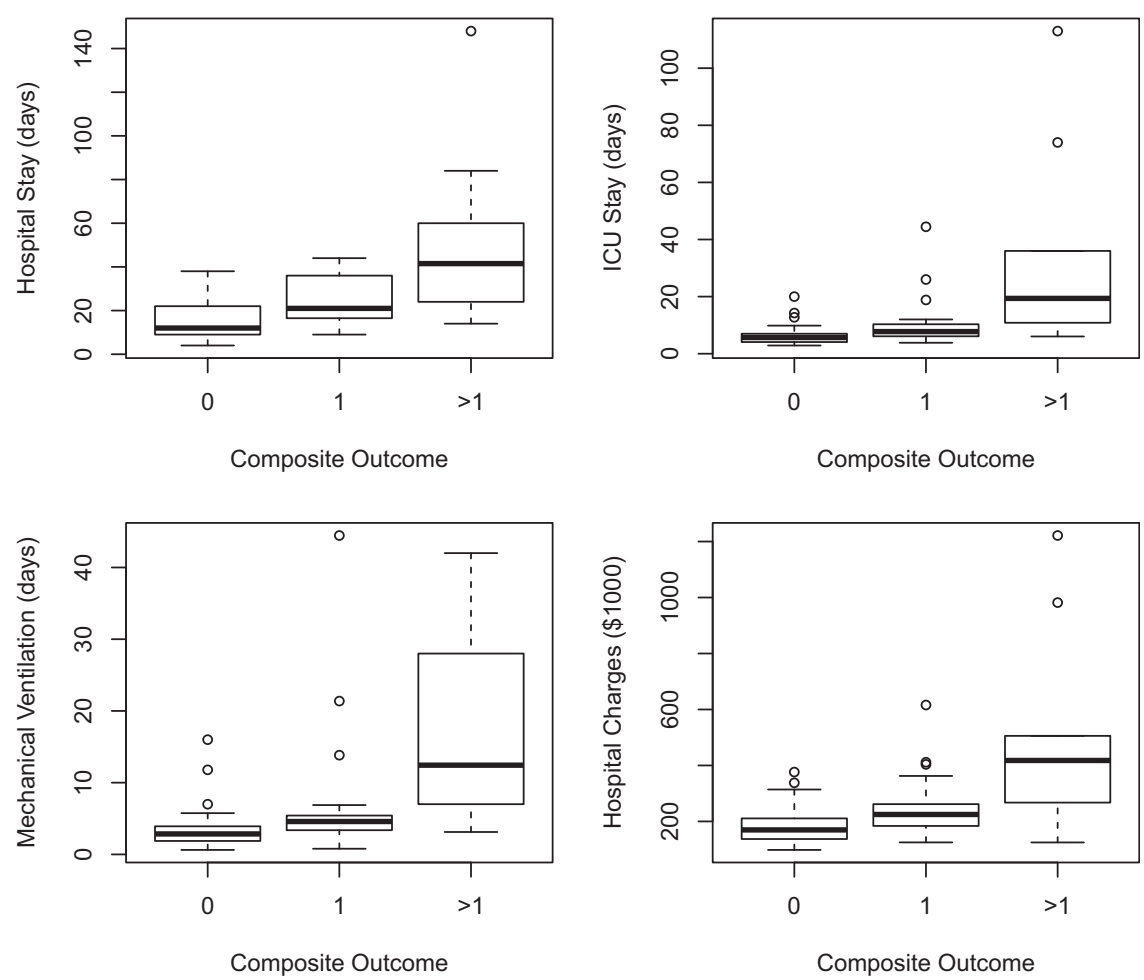

FIGURE 1. Composite outcome and postoperative outcomes. Box-whisper plot of postoperative outcomes and composite outcome. Box represents IQR. Dark bar represents median. Whiskers extend to range of outcome up to $1.5 \times$ the IQR. Circles represent outliers beyond $1.5 \times$ the IQR. $0=$ patients who met no composite outcome, 1 = patients who met only 1 composite outcome criteria, $>1=$ patients who met $\geq 2$ composite outcome criteria. There is a statistically significant increase in median hospital stay, median ICU stay, median mechanical ventilation, and median hospital charges between each category $(P<.01$, Table 4). ICU, Intensive care unit; $I Q R$, interquartile range.

previously described associations with poor short- or longterm outcomes after congenital cardiac surgery, and as such represent clinically significant outcomes. ${ }^{16,18-21}$

The use of a composite outcome for end points in clinical research is done frequently in cardiovascular research and multiple other fields of medicine. ${ }^{10,25,26}$ Composite outcomes have the advantage of increasing the event rate, which increases the power of a study population, as long as the composite outcome event rate is closer to $50 \%$

TABLE 5. Clinical or laboratory criteria of composite outcome met and postoperative outcomes

\begin{tabular}{|c|c|c|c|c|}
\hline & \multicolumn{3}{|c|}{ Composite outcome } & \multirow[b]{3}{*}{$\begin{array}{c}P \\
\text { value }\end{array}$} \\
\hline & \multicolumn{2}{|c|}{ Yes } & \multirow{2}{*}{$\begin{array}{c}\text { No } \\
(n=46)\end{array}$} & \\
\hline & Clinical $(n=9)$ & $\begin{array}{c}\text { Laboratory } \\
\text { only }(n=21)\end{array}$ & & \\
\hline Hospital stay (d) & 36.0 & $21.0(17.0-39.0)$ & $12.0(9.0-22.0)$ & $<.01$ \\
\hline ICU stay $(d)$ & $15.5(7.7-20.9)$ & $8.1(6.2-12.0)$ & $5.7(4.1-7.0)$ & $<.01$ \\
\hline $\begin{array}{l}\text { Mechanical } \\
\text { ventilation (d) }\end{array}$ & $8.6(5.8-19.0)$ & $4.7(3.8-6.9)$ & $2.9(1.9-3.9)$ & $<.01$ \\
\hline $\begin{array}{l}\text { Hospital charges } \\
(\$ 1000)\end{array}$ & 403 (208-506) & 245 (194-362) & $170(137-211)$ & $<.01$ \\
\hline
\end{tabular}

Values displayed are median (IQR). Clinical = patients who had CPR or ECMO, or died. Laboratory only $=$ patients who had hepatic injury, renal injury, or lactic acidosis. $I C U$, Intensive care unit. than any of the individual component event rates. The studied composite outcome had an event rate of $39 \%$, which is closer to $50 \%$ than the most common individual component, hepatic injury with a $22 \%$ event rate. Thus, the use of this composite outcome will increase the power of a study. The reduction in study population has the advantages of reduced costs, ability to perform investigations in a timely manner, and avoidance of an arbitrary choice between several outcomes.

Despite the advantages of composite outcomes, their use can be misleading. This may be particularly relevant when the components of the composite outcome vary in clinical importance. Studies demonstrate that the treatment effect is often biggest for the less important components and smallest for the most important component. ${ }^{11}$ This is consistent with the present study in which the lower morbidity components of the composite outcome (hepatic and renal insufficiency and lactic acidosis) occurred more often than the more significant components of death, CPR, or mechanical circulatory support. To help overcome this bias toward less important components, a recent report suggested that weighted composite indexes are superior measure end points in clinical trials. ${ }^{10}$ The results of our study indicate that the clinical outcomes of death, ECMO, or CPR should be weighted more than the laboratory 

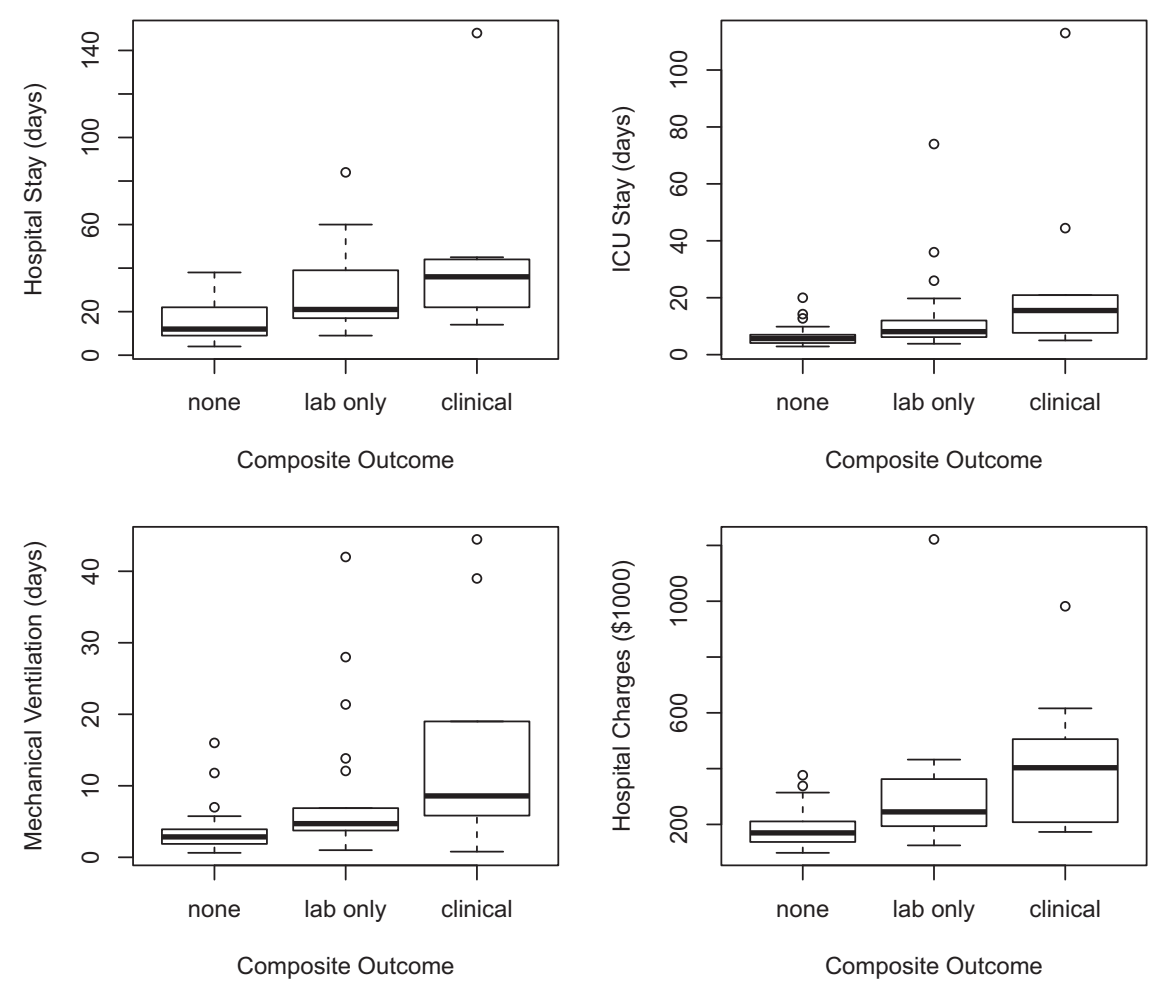

FIGURE 2. Clinical or laboratory criteria of composite outcome met and postoperative outcomes. Box-whisper plot of postoperative outcomes and whether clinical criteria, laboratory criteria only, or no composite outcome criteria were met. Box represents IQR. Dark bar represents median. Whiskers extend to range of outcome up to $1.5 \times$ the IQR. Circles represent outliers beyond $1.5 \times$ the IQR. None = patients who met no composite outcome, laboratory only $=$ patients who met only laboratory composite outcome criteria, clinical = patients who met any of the clinical composite outcome criteria (CPR, death, ECMO). There is a statistically significant increase in median hospital stay, ICU stay, duration of mechanical ventilation, and hospital charges between each category $(P<.01$, Table 5). $I C U$, Intensive care unit; $I Q R$, interquartile range; $C P R$, cardiopulmonary resuscitation; $E C M O$, extracorporeal membrane oxygenation.

outcomes. However, our study was not sufficiently powered to determine the individual weight that should be applied to each component of the composite outcome.

\section{Study Limitations}

The components of the composite outcome and cutoffs for liver injury, renal injury, and lactic acidosis were based on previous reports, and each component was given equal weight. The best methods to define hepatic injury, renal injury, elevated lactic acidosis, and the weight of each component should be studied and would likely require a large multi-institutional network. With a larger study population, cutoff levels for each laboratory measure would have been based on statistical analysis instead of previously used levels, and each component could have been weighted given its relative associations with outcomes. Only neonates were studied, so the results may not be applicable to other populations. The proposed composite outcome may not be a useful marker in less complex populations. The composite outcome was only compared with 4 other early outcome measures, and the relationship to other early and late outcomes warrants further investigation.

\section{CONCLUSIONS}

The ideal composite outcome in clinical research would have a relatively high prevalence, be composed of clinically meaningful components that are weighted appropriately, and correlate with other short and long-term outcomes. A composite outcome consisting of death, CPR, ECMO, elevated lactate, and hepatic and renal injury is easily measured, reproducible, and highly associated with important early operative outcomes in neonates undergoing cardiac operations with CPB. The studied composite outcome had a high prevalence rate, and its components were selected using prior evidence. This composite outcome could serve as a useful end point for future clinical studies. Future studies should further refine the studied composite outcome and determine whether the composite outcome is associated with long-term outcomes.

\section{References}

1. Khairy P, Ionescu-Ittu R, Mackie AS, Abrahamowicz M, Pilote L, Marelli AJ. Changing mortality in congenital heart disease. J Am Coll Cardiol. 2010;56: 1149-57.

2. Hovels-Gurich HH, Konrad K, Wiesner M, Minkenberg R, Herpertz-Dahlmann B, Messmer BJ, et al. Long term behavioural outcome after neonatal arterial switch operation for transposition of the great arteries. Arch Dis Child. 2002;87:506-10. 
3. Hovels-Gurich HH, Seghaye MC, Schnitker R, Wiesner M, Huber W, Minkenberg R, et al. Long-term neurodevelopmental outcomes in school-aged children after neonatal arterial switch operation. J Thorac Cardiovasc Surg. 2002;124:448-58

4. Ballweg JA, Wernovsky G, Gaynor JW. Neurodevelopmental outcomes following congenital heart surgery. Pediatr Cardiol. 2007;28:126-33.

5. Mahony L, Sleeper LA, Anderson PA, Gersony WM, McCrindle BW, Minich LL, et al. The Pediatric Heart Network: a primer for the conduct of multicenter studies in children with congenital and acquired heart disease. Pediatr Cardiol. 2006;27:191-8

6. Wernovsky G, Wypij D, Jonas RA, Mayer JE Jr, Hanley FL, Hickey PR, et al. Postoperative course and hemodynamic profile after the arterial switch operation in neonates and infants. A comparison of low-flow cardiopulmonary bypass and circulatory arrest. Circulation. 1995;92:2226-35.

7. Gaies MG, Gurney JG, Yen AH, Napoli ML, Gajarski RJ, Ohye RG, et al. Vasoactive-inotropic score as a predictor of morbidity and mortality in infants after cardiopulmonary bypass. Pediatr Crit Care Med. 2010;11:234-8.

8. Hoffman TM, Wernovsky G, Atz AM, Kulik TJ, Nelson DP, Chang AC, et al. Efficacy and safety of milrinone in preventing low cardiac output syndrome in infants and children after corrective surgery for congenital heart disease. Circulation. 2003;107:996-1002.

9. Butts RJ, Scheurer M, Atz AM, Zyblewski SC, Bradley SM, Hulsey TC, et al. Comparison of maximum vasoactive inotropic score and low cardiac output syndrome as markers of early postoperative outcomes after neonatal cardiac surgery. Pediatr Cardiol. 2012;33:633-8.

10. Bakal JA, Westerhout CM, Armstrong PW. Impact of weighted composite compared to traditional composite endpoints for the design of randomized controlled trials. Stat Methods Med Res. January 24, 2012 [Epub ahead of print]

11. Cordoba G, Schwartz L, Woloshin S, Bae H, Gotzsche PC. Definition, reporting, and interpretation of composite outcomes in clinical trials: systematic review. BMJ. 2010;341:c3920.

12. Moga MA, Manlhiot C, Marwali EM, McCrindle BW, Van Arsdell GS, Schwartz SM. Hyperglycemia after pediatric cardiac surgery: impact of age and residual lesions. Crit Care Med. 2011;39:266-72.

13. Costello JM, Polito A, Brown DW, McElrath TF, Graham DA, Thiagarajan RR, et al. Birth before 39 weeks' gestation is associated with worse outcomes in neonates with heart disease. Pediatrics. 2010;126:277-84.

14. Hsu JH, Keller RL, Chikovani O, Cheng H, Hollander SA, Karl TR, et al. B-type natriuretic peptide levels predict outcome after neonatal cardiac surgery. $J$ Thorac Cardiovasc Surg. 2007;134:939-45.
15. Graham EM, Atz AM, Butts RJ, Baker NL, Zyblewski SC, Deardorff RL, et al. Standardized preoperative corticosteroid treatment in neonates undergoing cardiac surgery: results from a randomized trial. J Thorac Cardiovasc Surg. 2011 142:1523-9.

16. Charpie JR, Dekeon MK, Goldberg CS, Mosca RS, Bove EL, Kulik TJ. Serial blood lactate measurements predict early outcome after neonatal repair or palliation for complex congenital heart disease. J Thorac Cardiovasc Surg. 2000;120:73-80.

17. Vazquez P, Lopez-Herce J, Carrillo A, Sancho L, Bustinza A, Diaz A. Hepatic dysfunction after cardiac surgery in children. Pediatr Crit Care Med. 2001;2: 44-50.

18. Toth R, Breuer T, Cserep Z, Lex D, Fazekas L, Sapi E, et al. Acute kidney injury is associated with higher morbidity and resource utilization in pediatric patients undergoing heart surgery. Ann Thorac Surg. 2012;93:1984-90.

19. Hansen G, Joffe AR, Nettel-Aguirre A, Robertson CM, Biggs WS, Ross DB et al. Two-year survival and neurodevelopmental outcomes after cardiopulmonary resuscitation in neonatal patients after complex cardiac surgery. Resuscita tion. 2011;82:313-8.

20. Flick RP, Sprung J, Gleich SJ, Barnes RD, Warner DO, Dearani JA, et al. Intraoperative extracorporeal membrane oxygenation and survival of pediatric patients undergoing repair of congenital heart disease. Paediatr Anaesth. 2008; 18:757-66.

21. Krawczeski CD, Goldstein SL, Woo JG, Wang Y, Piyaphanee N, Ma Q, et al. Temporal relationship and predictive value of urinary acute kidney injury biomarkers after pediatric cardiopulmonary bypass. J Am Coll Cardiol. 2011;58 2301-9.

22. Coccia R, Spadaccio C, Foppoli C, Perluigi M, Covino E, Lusini M, et al. The effect of simvastatin on erythrocyte membrane fluidity during oxidative stress induced by cardiopulmonary bypass: a randomized controlled study. Clin Ther. 2007;29:1706-17.

23. Siegel LB, Dalton HJ, Hertzog JH, Hopkins RA, Hannan RL, Hauser GJ. Initia postoperative serum lactate levels predict survival in children after open heart surgery. Intensive Care Med. 1996;22:1418-23.

24. Jenkins KJ, Gauvreau K, Newburger JW, Spray TL, Moller JH, Iezzoni LI. Consensus-based method for risk adjustment for surgery for congenital heart disease. J Thorac Cardiovasc Surg. 2002;123:110-8.

25. Srikrishna S, Robinson D, Cardozo L. Role of composite endpoints as an outcome assessment tool in urogenital prolapse. J Obstet Gynaecol. 2012;32:276-9.

26. Fitzgerald O, Helliwell P, Mease P, Mumtaz A, Coates L, Pedersen R, et al. Ap plication of composite disease activity scores in psoriatic arthritis to the PRESTA data set. Ann Rheum Dis. 2012;71:358-62. 